\section{GW23-e2186 APPLICATIONS OF EMERGENT PERCUTANEOUS CORONARY INTERVENTION COMBINED WITH INTRA- AORTIC BALLOON PUMP ON INTENSIVE ACUTE MYOCARDIAL INFARCTION}

doi:10.1136/heartjnl-2012-302920l.7
Results Among the 57 patients included in the group received IABP, $38(67.2 \%)$ were male and the mean age was $62.75 \pm 11.96$ years and 20 (34.5\%) had cardiogenic shock, 37 (64.9\%) had acute myocardial infarction, 25 (43.9\%) had hypertension, 21 (36.8\%) had diabetes mellitus, 31 (54.4\%) had smoking, 13 (22.8\%) had hyperlipidaemia, $16(28.1 \%)$ had previous PCI. Among the 48 patients included in the non-IABP group, which was $32(66.7 \%), 56.81 \pm 9.75$ years, 6 (12.5\%), 6 (12.5\%), 27 (56.3\%), 17 (35.4\%), 25 (52.1\%), 6 (12.5\%), 4 (8.3\%), respectively. After the PCI operation, the incidence rate of postoperative complications and the incidence rate of cardiovascular events 12 months after the operation in the group received IABP were obviously lower than those in the group which did not receive the IABP $(p<0.05)$, include death $\left(x^{2}=13.625, p=0.000\right)$, cardiogenic shock $\left(x^{2}=6.751, p=0.009\right)$, revascularisation $(x 2=6.151$, $\mathrm{p}=0.013)$, re-hospitalisation $(x 2=7.027, \mathrm{p}=0.008)$.

Conclusions The treatment of IABP on patients with intensive AMI can provide the active hemodynamic support, improve cardiac function and decrease the occurrence rate of cardiac events, is safe and effective, and is valuable for general application.

Objectives Intra-aortic balloon pump plays an important role in hemodynamic instability following acute myocardial infarction undergoing percutaneous coronary intervention. It improves diastolic coronary and systemic blood flow and increases cardiac work. The purpose of this study was to review the application value of the emergency percutaneous coronary intervention (PCI) combined with intra-aortic balloon pump (IABP) in treatment of patients with intensive acute myocardial infarction (AMI).

Methods The data of the intensive patients with AMI hospitalised through emergency in our heart centre from 2009 to 2011 were collected. Among these patients, 105 cases were treated with emergent PCI and they were divided into two groups according to whether they were given IABP treatment. The clinical data, vascular lesion and the cardiovascular events 12 months after the operation were analysed and compared between the two groups. Measurement data are expressed as means $\pm \mathrm{SD}$ with the $t$ test, and count data with the $\mathrm{x} 2$ test. 